\title{
CONTROL DINÁMICO DE MANIPULADORES MÓVILES CON REALIMENTACIÓN VISUAL
}

\author{
José L. Ramón, Álvaro Belmonte, Jorge Pomares, Gabriel J. García, Carlos A. Jara, Andrés Úbeda \\ Departamento de Física, Ingeniería de Sistemas y Teoría de la Señal \\ Universidad de Alicante \\ \{jl.ramon@,bb35@alu.,jpomares@,gjgg@, carlos.jara@, andres.ubeda@\}ua.es
}

Ester Abad, María Ángeles Rueda

Unidad de Daño Cerebral Adquirido

Hospital de San Vicente del Raspeig

\{abad_est, rueda_marang\}@gva.es

\begin{abstract}
Resumen
Este articulo propone un sistema de control visual directo basado en imagen para guiar a un manipulador móvil. El robot está compuesto por una plataforma móvil diferencial con un brazo de $7 \mathrm{gdl}$ (grados de libertad) y una cámara en configuración eye-in-hand. Como se describe en el documento, la información visual se emplea para guiar tanto el robot manipulador como la plataforma base durante el seguimiento de trayectorias definidas en el plano imagen. A diferencia de los enfoques anteriores, el sistema propuesto en este documento emplea un control visual directo para guiar al manipulador móvil. De esta forma, el controlador genera directamente los pares (fuerza y momentos) que se aplicarán al manipulador y la plataforma base teniendo en cuenta la dinámica del robot. Este artículo describe los diferentes componentes cinemáticos y dinámicos del controlador propuesto y se valida a partir del seguimiento de trayectorias de imágenes repetitivas y no repetitivas.
\end{abstract}

Palabras clave: control visual, control basado en imagen, manipulador móvil, control directo

\section{INTRODUCCIÓN}

Hoy en día, los nuevos robots manipuladores industriales incluyen estructuras cinemáticas redundantes que le permiten realizar tareas complejas en entornos no estructurados $\mathrm{y} / \mathrm{o}$ dinámicos. Esta redundancia proporciona a estos robots una gran cantidad de gdl a partir de estructuras articulares complejas. Este es el caso de los manipuladores móviles que incluyen un brazo robot sobre una base móvil. Estas estructuras superan las limitaciones intrínsecas de la construcción mecánica y proporcionan más flexibilidad durante el desempeño de las tareas. Sin embargo, controlar un sistema redundante durante este tipo de tareas implica no sólo controlar el movimiento correcto del efector final, sino también el movimiento correcto para la estabilización de la articulación. Este artículo presenta un nuevo controlador visual directo basado en imagen para el guiado de manipuladores móviles donde la tarea a realizar se especifica en el espacio de la imagen. Los controladores visuales clásicos basados en imagen determinan las velocidades de cámara necesarias para posicionar el robot con respecto a un objeto de referencia [2]. Usando este último enfoque, el controlador interno del robot calcula los pares articulares para alcanzar las velocidades de cámara mencionadas anteriormente. Sin embargo, por medio del control visual directo, se elimina el bucle de control interno de los servomotores y el control visual se emplea para estabilizar directamente el robot [9]. El resultado es un control más rápido y más preciso que reacciona más rápidamente ante cambios abruptos en las trayectorias de la imagen.

El control de los sistemas mecánicos redundantes ha sido un tema de investigación relevante en los últimos años, donde muchos autores han propuesto diferentes algoritmos de control basados en la dinámica del robot. Vale la pena señalar el trabajo presentado por Khatib [4], que propuso un esquema de control directo para robots redundantes utilizando la pseudoinversa de la matriz Jacobiana. Otros trabajos como los presentados por Xian [13] y Zergeroglu [14] se basan en un controlador no lineal que garantiza un seguimiento asintótico en el espacio Cartesiano. Tatlicioglu [11] y Lin [7] propusieron aplicar otro tipo de controladores, como el control adaptativo y óptimo en la planificación de la trayectoria. Otros enfoques recientes se centran en las redes neuronales debido a sus capacidades de aprendizaje y computación paralela. En este campo, la mayoría de las contribuciones tratan de compensar tanto las no linealidades [8] como las incertidumbres estructuradas y no estructuradas del sistema [5]. Los enfoques 
comentados anteriormente se basan en el control de la posición/orientación y/o el control de velocidad. Sin embargo, el controlador propuesto en este artículo emplea información de imagen para el guiado del robot. El uso de este sistema de control visual permite el seguimiento de formas complejas en el espacio de la imagen utilizando el manipulador móvil, garantizando que se obtenga el movimiento articular correcto.

Dentro del campo del control visual indirecto, se pueden encontrar varios enfoques para el guiado de robots móviles o manipuladores móviles. En [1], se propone un enfoque $2 \mathrm{D}$ basado en la matriz de homografía para el control visual de un manipulador móvil que no requiere ninguna medida de la estructura 3D del objetivo observado. En [10] se propone un sistema de detección y control del espacio de tareas diseñado para controlar el movimiento del efector final de un manipulador móvil en presencia de un movimiento de la base dinámico y desconocido. En [3], se propone un enfoque de control visual basado en imagen con resolución de redundancia para el guiado de un manipulador móvil. En [6] se describe un enfoque de control visual indirecto basado en posición para este mismo fin. En [12], se desarrolló un esquema de control que utiliza control visual basado en imagen junto con una plataforma de manipulador móvil funcional, donde se integró en el sistema una configuración de cámara híbrida compuesta por cámaras de visión monocular y estéreo. En estos enfoques indirectos previos, el controlador asume que el dispositivo guiado es un sistema de posicionamiento perfecto $\mathrm{y}$, por lo tanto, no se considera su dinámica. Sin embargo, en el enfoque propuesto en este artículo, el uso de un sistema de control visual directo permite tener en cuenta la dinámica del manipulador y la base.

El artículo se divide en las siguientes secciones. Primero, la Sección 2 describe el sistema robótico y los sistemas de referencia principales. La dinámica del sistema se describe en la Sección 3, y la formulación del controlador se detalla en la Sección 4. La Sección 5 describe las principales relaciones cinemáticas empleadas en la formulación del controlador. Finalmente, en la Sección 6 se describen los principales resultados obtenidos en la aplicación del controlador propuesto.

\section{ARQUITECTURA DEL SISTEMA}

La Figura 1 representa los principales componentes del manipulador móvil (el robot TIAGo de PAL Robotics). El robot está compuesto de una plataforma móvil diferencial con un brazo de 7 gdl, una cámara RGB-D que no se emplea en este artículo, y una cámara adicional situada en configuración eye-inhand. El sistema de referencia $\mathcal{F}_{p}$ está localizado en la plataforma del robot móvil, mientras que $\mathcal{F}_{m}$ es el sistema de referencia del manipulador fijado en la base del brazo robótico. El sistema de referencia del mundo se llama $\mathcal{F}_{o}$. El sistema de referencia del efector final del manipulador móvil, $\mathcal{F}_{e}$, se sitúa en el efector final del brazo robótico, y el sistema de referencia $\mathcal{F}_{c}$ es sistema de referencia de la cámara. En este artículo se considera un sistema en configuración eye-in-hand donde $\mathcal{F}_{c}=\mathcal{F}_{e}$. La tabla 1 muestra la notación de las transformaciones entre los distintos sistemas de referencia utilizados en este artículo.
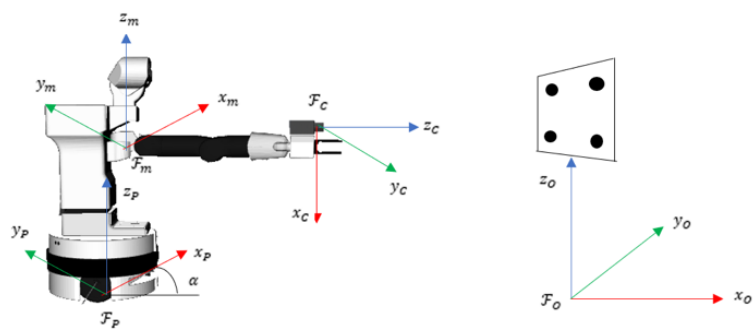

Figura 1: Componentes del sistema

Tabla 1: Notación.

\begin{tabular}{|c|c|}
\hline Variable & Definición \\
\hline$\left(x_{p m}, y_{p m}, z_{p m}\right)$ & $\begin{array}{c}\text { Translación de } \mathcal{F}_{m} \text { con respecto al } \\
\text { sistema de referencia } \mathcal{F}_{p}\end{array}$ \\
\hline$\left(x_{o p}, y_{o p}, z_{o p}\right)$ & Posición de $\mathcal{F}_{p}$ con respecto a $\mathcal{F}_{o}$ \\
\hline$\alpha$ & $\begin{array}{c}\text { Ángulo entre el eje } \mathrm{X} \text { del sistema } \mathcal{F}_{p} \mathrm{y} \\
\text { el eje } \mathrm{X} \text { del sistema } \mathcal{F}_{o}\end{array}$ \\
\hline$\left(x_{m e}, y_{m e}, z_{m e}\right)$ & Posición de $\mathcal{F}_{e}$ con respecto a $\mathcal{F}_{m}$ \\
\hline$\left(\phi_{x m e}, \phi_{y m e}, \phi_{z m e}\right)$ & Orientación de $\mathcal{F}_{e}$ con respecto a $\mathcal{F}_{m}$ \\
\hline
\end{tabular}

Con $\boldsymbol{q}_{m}^{T} \in \mathfrak{R}^{\mathrm{m}} \quad$ se representan las coordenadas articulares generalizadas del manipulador (en nuestro caso, $\mathrm{m}=7)$ y $\boldsymbol{q}_{p}^{T} \in \mathfrak{R}^{\mathrm{np}}$ son las coordenadas generalizadas de la plataforma. Las coordenadas generalizadas de manipulador y plataforma se pueden representar como $\boldsymbol{q}=\left[\begin{array}{ll}\boldsymbol{q}_{p}^{T} & \boldsymbol{q}_{m}^{T}\end{array}\right]$. Los comandos del motor se consideran como un conjunto de velocidades de comando de la plataforma, $\boldsymbol{u}_{p} \in \mathbb{R}^{\mathrm{p}}$, y velocidades de comando del manipulador, $\boldsymbol{u}_{m} \in \mathbb{R}^{\mathrm{m}}$, así:

$$
\boldsymbol{u}=\left[\begin{array}{ll}
\boldsymbol{u}_{p}^{T} & \boldsymbol{u}_{m}^{T}
\end{array}\right]^{T}
$$

En esta ecuación las acciones de control para el motor del manipulador son las velocidades articulares, i. e.

$\dot{\boldsymbol{q}}_{m}=\boldsymbol{u}_{m}$, y a partir del modelo cinemático de la plataforma móvil se puede considerar la siguiente relación:

$$
\dot{\boldsymbol{q}}_{p}=\boldsymbol{\psi}\left(\boldsymbol{q}_{p}\right) \boldsymbol{u}_{p}
$$


donde $\boldsymbol{u}_{p}=\left[\begin{array}{ll}v & \omega\end{array}\right]^{T}$ indica las velocidades lineal y angular de la plataforma, y la función $\boldsymbol{\psi}$ representa el espacio de velocidad admisible en cada configuración de la plataforma móvil.

Se ha elegido una cámara en configuración eye-inhand que observa un conjunto de cuatro características visuales ubicadas en la superficie del objeto a seguir. Como muestra la Figura 1, se ha añadido al objeto a seguir un patrón blanco con cuatro puntos negros. Por lo tanto, el sistema de visión artificial extrae cuatro puntos de características visuales, $\boldsymbol{s}=\left[\boldsymbol{f}_{1}, \boldsymbol{f}_{2}, \ldots, \boldsymbol{f}_{k}\right]^{\mathrm{T}}$, que corresponden al centro de estos puntos negros $(\mathrm{k}=4$ en la Figura 1$)$.

\section{DINÁMICA DEL SISTEMA}

Esta sección describe las principales ecuaciones dinámicas para el sistema robótico. Las ecuaciones dinámicas de movimiento del manipulador móvil se pueden escribir como:

$$
\left[\begin{array}{c}
\boldsymbol{F}_{b} \\
\boldsymbol{\tau}
\end{array}\right]=\left[\begin{array}{ll}
\boldsymbol{M}_{b b} & \boldsymbol{M}_{b m} \\
\boldsymbol{M}_{b m}^{\mathrm{T}} & \boldsymbol{M}_{m m}
\end{array}\right]\left[\begin{array}{c}
\dot{\boldsymbol{u}}_{p} \\
\ddot{\boldsymbol{q}}_{m}
\end{array}\right]+\left[\begin{array}{c}
\boldsymbol{c}_{b} \\
\boldsymbol{c}_{m}
\end{array}\right]
$$

donde:

- $\quad \ddot{\boldsymbol{q}}_{m} \in \mathfrak{R}^{m}$ es el conjunto de aceleraciones articulares del robot manipulador.

- $\quad \dot{\boldsymbol{u}}_{p}=\left[\begin{array}{ll}\dot{v} & \dot{\omega}\end{array}\right]^{T}$ son las aceleraciones lineal $\mathrm{y}$ angular de la plataforma.

- $\quad \boldsymbol{M}_{b b} \in \mathfrak{R}^{p \times p}$ es la matriz de inercia de la plataforma.

- $\quad \boldsymbol{M}_{b m} \in \mathfrak{R}^{p \times m}$ es la matriz de inercia acoplada de la base y el manipulador.

- $\boldsymbol{M}_{m m} \in \mathfrak{R}^{m \times m}$ es la matriz de inercia del manipulador.

- $\boldsymbol{c}_{b}$ y $\boldsymbol{c}_{m}$ son términos no lineales dependientes de la velocidad/desplazamiento de la base y el manipulador, respectivamente.
- $\quad \boldsymbol{F}_{b}$ es la fuerza y momentos ejercidos en la base.

- $\boldsymbol{\tau}$ es el par articular aplicado en el robot manipulador.

El movimiento del manipulador móvil se rige por los pares aplicados en las articulaciones del manipulador y por la fuerza y el momento ejercidos sobre la base. Por lo tanto, la ecuación (3) se puede escribir en la siguiente forma:

$$
\widetilde{\boldsymbol{\tau}}=\widetilde{\boldsymbol{M}} \boldsymbol{u}+\widetilde{\boldsymbol{C}}
$$

donde $\tilde{\boldsymbol{\tau}}=\left[\begin{array}{c}\boldsymbol{F}_{\mathrm{b}} \\ \boldsymbol{\tau}\end{array}\right], \tilde{\boldsymbol{M}}=\left[\begin{array}{ll}\boldsymbol{M}_{b b} & \boldsymbol{M}_{b m} \\ \boldsymbol{M}_{b m}^{\mathrm{T}} & \boldsymbol{M}_{m m}\end{array}\right], \dot{\boldsymbol{u}}=\left[\begin{array}{c}\dot{\boldsymbol{u}}_{p} \\ \ddot{\boldsymbol{q}}_{m}\end{array}\right], \mathrm{y}$ $\widetilde{\boldsymbol{C}}=\left[\begin{array}{l}\boldsymbol{c}_{b} \\ \boldsymbol{c}_{m}\end{array}\right]$.

\section{CONTROL VISUAL DEL MANIPULADOR MÓVIL}

Esta sección describe el controlador propuesto para el guiado de la plataforma móvil. La Figura 2 representa los componentes principales del sistema de control propuesto. Para describir estos componentes, primero, se define $\ddot{\boldsymbol{s}}_{r}$ como las aceleraciones de las referencias visuales en la imagen que serán empleadas por el sistema de control visual. El valor de esta referencia visual se describe considerando el sistema de cámara en configuración eye-in-hand, que extrae un conjunto de $\mathrm{k}$ puntos de características de imagen. Esta referencia viene dada por la siguiente ecuación en el espacio de la imagen:

$$
\left(\ddot{\boldsymbol{s}}_{d}-\ddot{\boldsymbol{s}}\right)+\boldsymbol{K}_{D}\left(\dot{\boldsymbol{s}}_{d}-\dot{\boldsymbol{s}}\right)+\boldsymbol{K}_{P}\left(\boldsymbol{s}_{d}-\boldsymbol{s}\right)=0
$$

donde $\ddot{\boldsymbol{s}}_{d}, \dot{\boldsymbol{s}}_{d}$ y $\boldsymbol{s}_{d}$ son las aceleraciones, velocidades y posiciones deseadas de las características visuales en el espacio de la imagen, respectivamente. $\boldsymbol{K}_{P}$ y $\boldsymbol{K}_{D}$ son matrices de ganancia proporcional y derivada,

Cinemática y dinámica del robot

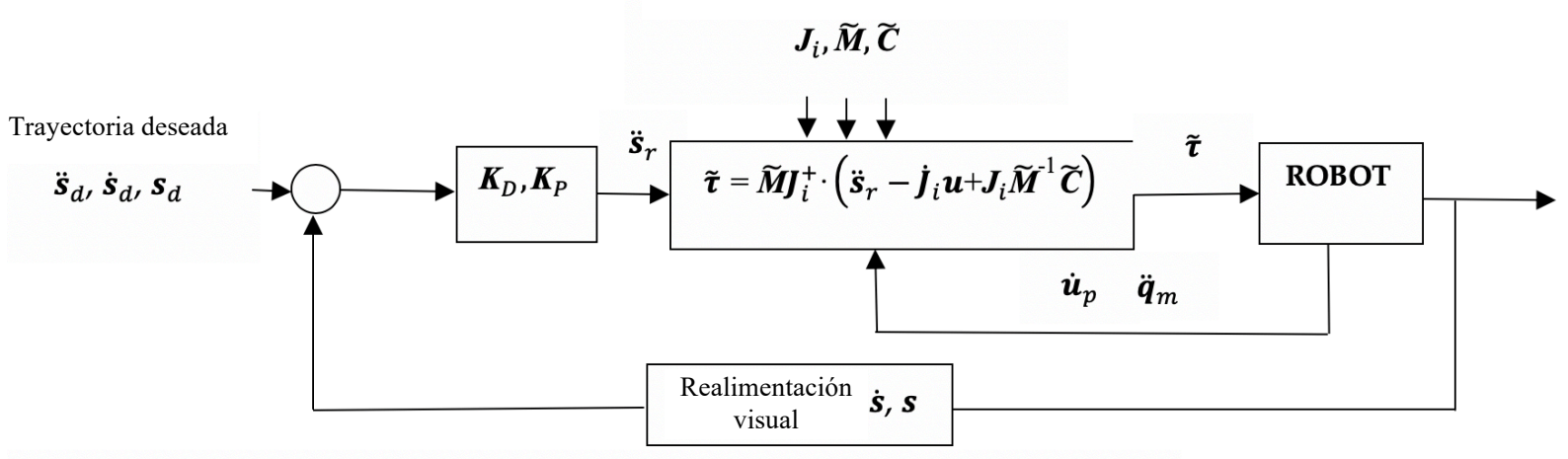

Figura 2: Esquema de control del manipulador móvil 
respectivamente. Esta ecuación se puede expresar con respecto al error en la imagen de la siguiente manera:

$$
\ddot{\boldsymbol{s}}_{d}+\boldsymbol{K}_{D} \dot{\boldsymbol{e}}_{s}+\boldsymbol{K}_{P} \boldsymbol{e}_{s}=\ddot{\boldsymbol{s}}_{r}
$$

donde $\boldsymbol{e}_{s}$ y $\dot{\boldsymbol{e}}_{s}$ son el error en la imagen y la derivada temporal del error en la imagen, respectivamente. Como se indica, la variable $\ddot{\boldsymbol{s}}_{r}$ denota las aceleraciones de las características visuales del controlador basado en imagen propuesto. Por lo tanto, a partir de la dinámica inversa, se propone el siguiente controlador basado en imagen:

$$
\tilde{\boldsymbol{\tau}}=\widetilde{\boldsymbol{M}} \boldsymbol{J}_{i}^{+} \cdot\left(\ddot{\boldsymbol{s}}_{d}+\boldsymbol{K}_{D} \dot{\boldsymbol{e}}_{s}+\boldsymbol{K}_{P} \boldsymbol{e}_{s}-\dot{\boldsymbol{J}}_{i} \boldsymbol{u}+\boldsymbol{J}_{i} \widetilde{\boldsymbol{M}}^{-1} \widetilde{\boldsymbol{C}}\right)
$$

donde, $\boldsymbol{J}_{i}$, es la Jacobiana de la imagen que relaciona las velocidades de comando de los motores $\boldsymbol{u}$ y la derivada respecto al tiempo del conjunto de características visuales $\boldsymbol{s}$.

$$
\dot{\boldsymbol{s}}=\boldsymbol{J}_{i} \boldsymbol{u}
$$

Otra relación cinemática útil para la definición del controlador propuesto es la aceleración de las características en la imagen, o la segunda derivada respecto al tiempo de $s$. Esta relación se obtiene mediante la definición de $\boldsymbol{J}_{i}$ y diferenciando respecto al tiempo:

$$
\ddot{\boldsymbol{s}}=\boldsymbol{J}_{i} \dot{\boldsymbol{u}}+\dot{\boldsymbol{J}}_{i} \boldsymbol{u}
$$

El comportamiento en bucle cerrado se calcula a partir de la ecuación (4) como:

$$
\begin{gathered}
\widetilde{\boldsymbol{M}} \dot{\boldsymbol{u}}+\widetilde{\boldsymbol{C}}= \\
\widetilde{\boldsymbol{M}} \boldsymbol{J}_{i}^{+} \cdot\left(\ddot{\boldsymbol{s}}_{d}+\boldsymbol{K}_{D} \dot{\boldsymbol{e}}_{s}+\boldsymbol{K}_{P} \boldsymbol{e}_{s}-\dot{\boldsymbol{J}}_{i} \boldsymbol{u}+\boldsymbol{J}_{i} \widetilde{\boldsymbol{M}}^{-1} \widetilde{\boldsymbol{C}}\right)
\end{gathered}
$$

Para demostrar el seguimiento asintótico de la referencia de la imagen, la ecuación (10) se puede simplificar multiplicando previamente sus lados izquierdo y derecho por $\boldsymbol{J}_{i} \widetilde{\boldsymbol{M}}^{-1}$ :

$$
\boldsymbol{J}_{i} \dot{\boldsymbol{u}}=\ddot{\boldsymbol{s}}_{d}+\boldsymbol{K}_{D} \dot{\boldsymbol{e}}_{s}+\boldsymbol{K}_{P} \boldsymbol{e}_{s}-\dot{\boldsymbol{J}}_{i} \boldsymbol{u}
$$

Finalmente, usando la relación expresada en la Ecuación (9), se puede concluir que:

$$
\ddot{\boldsymbol{e}}_{S}=-\boldsymbol{K}_{D} \dot{\boldsymbol{e}}_{S}-\boldsymbol{K}_{P} \boldsymbol{e}_{s}
$$

Por lo tanto, cuando $\boldsymbol{J}_{i}$ es de rango completo, el controlador visual expresado en la Ecuación (7) logra un seguimiento asintótico de una trayectoria definida en la imagen.

\section{JACOBIANA DE LA IMAGEN}

Esta sección describe la formulación jacobiana y cine- mática de la imagen requerida para la definición del controlador propuesto. Como se indicó anteriormente, el sistema de visión artificial extrae $\mathrm{k}$ puntos de características visuales del objeto observado $\boldsymbol{s}=$ $\left[\boldsymbol{f}_{1} \boldsymbol{f}_{2}, \ldots, \boldsymbol{f}_{k}\right]^{\mathrm{T}} \in \mathbb{R}^{\mathrm{k}}$. El controlador visual directo basado en imagen debe realizar el guiado del manipulador móvil para el seguimiento de la trayectoria deseada en el espacio de la imagen, $\boldsymbol{s}_{\mathrm{d}}(t)$. El enfoque de control visual permite el control de las articulaciones y la base del manipulador utilizando estas $\mathrm{k}$ características visuales, utilizando una configuración eye-in-hand, donde el efector final del manipulador sujeta la cámara.

Considerando una de las características visuales, $\boldsymbol{f}_{i}$, la relación entre las velocidades en el espacio de la imagen, $\dot{\boldsymbol{f}}_{i}$, y la velocidad en el espacio Cartesiano de la cámara (expresada en su propio sistema de referencia), $\dot{\boldsymbol{r}}_{\mathrm{c}}=\left[\begin{array}{ll}\boldsymbol{v}_{c} & \boldsymbol{\omega}_{c}\end{array}\right]^{T}$, viene dada por:

$$
\dot{\boldsymbol{f}}_{i}=\boldsymbol{J}_{f i}\left(\boldsymbol{f}_{i,}, Z_{i}\right)\left[\begin{array}{l}
\boldsymbol{v}_{c} \\
\boldsymbol{\omega}_{c}
\end{array}\right]
$$

donde $Z_{i}$ es la profundidad de la característica de la imagen y el valor de $\boldsymbol{J}_{f i}$ se puede encontrar en [2]. En este documento, se empleará una aproximación de control visual basado en imagen para el guiado del manipulador móvil, por lo tanto, se debe definir una Jacobiana de la imagen, $\boldsymbol{J}_{i}$, que relaciona los comandos del motor $\boldsymbol{u}$ y la derivada respecto al tiempo del conjunto de características de la imagen $\boldsymbol{s} . \boldsymbol{J}_{i}$ se ha definido como el producto de las matrices $\boldsymbol{J}_{s}$ y $\boldsymbol{J}_{c}$. Por lo tanto, $\boldsymbol{J}_{i}=\boldsymbol{J}_{s} \boldsymbol{J}_{c}$. El valor de estas matrices se indica en la Figura 3.

Como puede verse en la Figura 3, la matriz de interacción $\boldsymbol{J}_{S} \in \mathbb{R}^{2 \mathrm{k} \times 6}$ para el conjunto de puntos de características de imagen, $\boldsymbol{s}$, es la pila de k matrices $\boldsymbol{J}_{f i}$ para cada característica $\left(\boldsymbol{Z}=\left[\begin{array}{lll}Z_{1} & \ldots & Z_{k}\end{array}\right]^{T} \in \mathbb{R}^{\mathrm{k}}\right)$. La matriz $\boldsymbol{J}_{c}$ relaciona la velocidad del efector final con respecto al sistema de referencia de la cámara $\left[\begin{array}{ll}\boldsymbol{v}_{c} & \boldsymbol{\omega}_{c}\end{array}\right]^{T}$ y los comandos del motor $\boldsymbol{u}$. En la Figura 3 , también se indica el valor de $\boldsymbol{J}_{c}$. En esta figura, el Jacobiano del robot, $\boldsymbol{J}_{6 \times 6}$, se utiliza para relacionar las velocidades articulares y las velocidades Cartesianas. Adicionalmente, se emplea la matriz de rotación $\boldsymbol{R}_{c}$. Esta matriz representa la orientación del sistema $\mathcal{F}_{c}$ con respecto al sistema $\mathcal{F}_{o}$.

\section{RESULTADOS}

Esta sección presenta el comportamiento del sistema durante el seguimiento de diferentes trayectorias. La Sección 6.1 describe el comportamiento del sistema durante el seguimiento de trayectorias definidas en la imagen que sólo requieren el uso del brazo robot. 


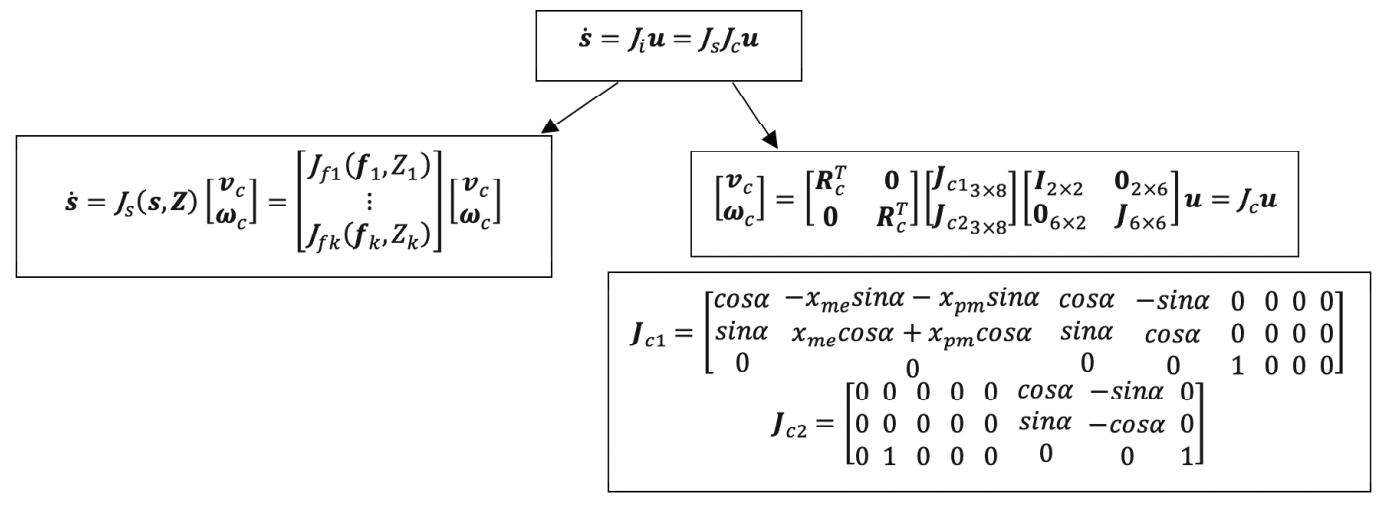

Figura 3: Formulación de la Jacobiana de la imagen

Además, la Sección 6.2 muestra diferentes experimentos de seguimiento que requieren el movimiento tanto del brazo del robot como de la plataforma base. El robot es guiado por un sistema de cámara eye-in-hand. Se consideran los parámetros de una cámara Gigabit Ethernet TM6740GEV, que adquiere 200 imágenes por segundo con una resolución de 1280x1024 píxeles.

\subsection{TRAYECTORIAS DE SEGUIMIENTO UTILIZANDO SOLO EL BRAZO ROBOT}

Esta sección describe el comportamiento del sistema durante el seguimiento de una trayectoria repetitiva. Sólo se emplea un punto como característica visual en este experimento y el seguimiento se realiza teniendo en cuenta únicamente los movimientos del brazo robot (el seguimiento no requiere el movimiento de la plataforma base). La trayectoria deseada se define por:

$$
\begin{gathered}
s_{d}=\left[\begin{array}{l}
f_{x d} \\
f_{y d}
\end{array}\right]= \\
{\left[\begin{array}{l}
320+166 \text { os }(\omega t+\pi / 4)+20 \sin (\omega 5 t) \\
265+160 \sin (\omega t+\pi / 4)+15 \sin (\omega 5 t)
\end{array}\right]}
\end{gathered}
$$

La Figura 4 muestra la trayectoria de imagen deseada (rojo) y la obtenida utilizando el sistema de control visual directo basado en imagen propuesto. Además, la tabla 2 representa el error medio en píxeles durante

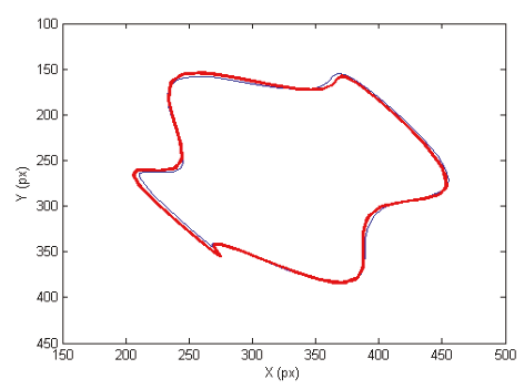

Figura 4: Trayectoria de imagen deseada (rojo) y trayectoria en imagen obtenida (azul) el seguimiento de la trayectoria de la imagen considerando diferentes velocidades de seguimiento, $\omega$. Como se puede ver en estas figuras, el seguimiento se realiza correctamente teniendo en cuenta sólo el movimiento del brazo.

Tabla 2: Error medio en la imagen durante el seguimiento de la trayectoria de imagen.

\begin{tabular}{|c|c|c|c|}
\hline & $\begin{array}{c}\boldsymbol{\omega}=\mathbf{0 . 5} \\
\mathbf{r a d} / \mathbf{s}\end{array}$ & $\begin{array}{c}\boldsymbol{\omega}=\mathbf{1} \\
\mathbf{r a d} / \mathbf{s}\end{array}$ & $\begin{array}{c}\boldsymbol{\omega}=\mathbf{2} \\
\mathbf{r a d} / \mathbf{s}\end{array}$ \\
\hline $\begin{array}{c}\text { Error medio en } \\
\text { imagen }\end{array}$ & $2.5 \mathrm{px}$ & $3.1 \mathrm{px}$ & $4.1 \mathrm{px}$ \\
\hline
\end{tabular}

\subsection{TRAYECTORIAS DE SEGUIMIENTO UTILIZANDO EL MANIPULADOR MÓVIL}

Esta sección detalla tres experimentos diferentes que requieren el movimiento tanto del brazo como de la plataforma base.

La Figura 5(a) y la Figura 5(b) representan la configuración inicial empleada en todos los experimentos. La Figura 5(a) representa el objetivo y el manipulador móvil con el sistema de cámara eye-inhand. La posición inicial de las características visuales se representa en la Figura 5(b). La trayectoria deseada empleada en el primer experimento es una trayectoria lineal que representa una traslación lateral de 200 px en las direcciones $\mathrm{X}$ e $\mathrm{Y}$ en la imagen desde la posición inicial de las características visuales. Como se puede ver en la Figura 6(b), el controlador visual basado en imagen realiza correctamente el seguimiento y se obtiene una línea recta en el espacio de la imagen. La Figura 6(a) representa la posición y orientación finales del manipulador móvil. El segundo experimento, representado en la Figura 7(a) y la Figura 7(b), evalúa el seguimiento cuando se debe realizar un desplazamiento en profundidad. Como puede verse en la Figura 7(a), el desplazamiento en profundidad se lleva a cabo y una vez que finaliza el experimento, el manipulador móvil está más cerca del 
(a)

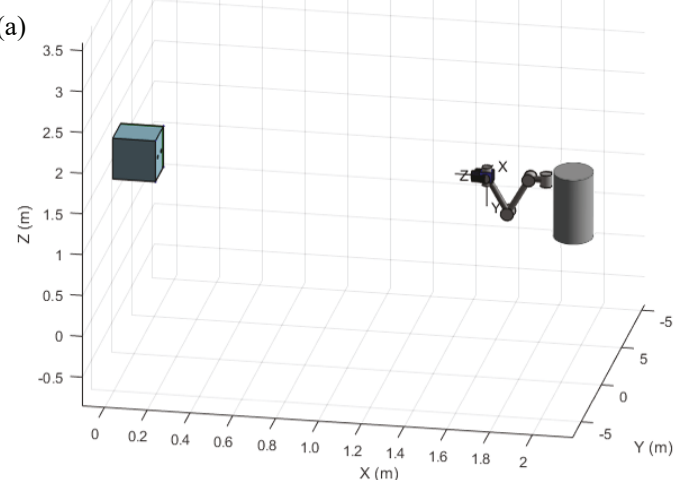

(b)

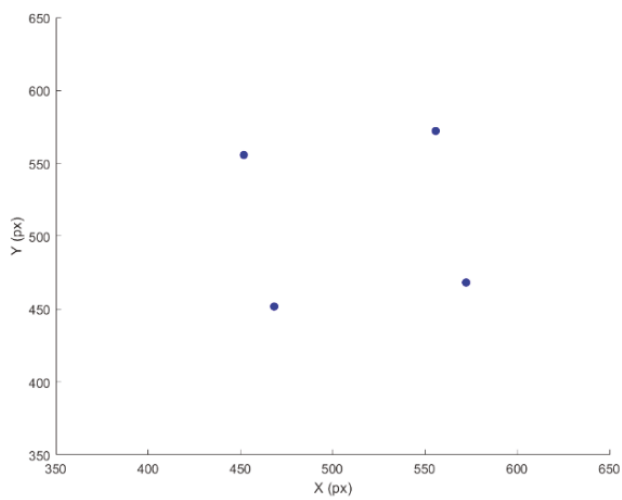

Figura 5: Posición del robot y trayectorias en la imagen en los experimentos: (a) posición inicial 3D del manipulador móvil y objeto, (b) características visuales iniciales extraidas por la cámara

objetivo. La Figura 7(b) representa la trayectoria de la imagen obtenida. Esta trayectoria corresponde a la trayectoria deseada especificada como un aumento lineal en profundidad desde la configuración de imagen inicial.

La Figura 8 representa los pares articulares aplicados al manipulador $(\mathrm{m}=1 \ldots 7)$ y la fuerza y momentos aplicados a la plataforma del robot durante el primer experimento. Como se puede ver en la Figura 8, tanto los pares, como la fuerza y el momento permanecen bajos y se obtiene un comportamiento suave durante el seguimiento.

Para el segundo experimento, la Figura 9 representa los pares articulares aplicados al manipulador y la fuerza y el momento aplicados a la plataforma base durante el movimiento del robot en profundidad. Cabe resaltar que, la reducción de la distancia entre los dos cuerpos (objetivo y robot) se obtiene modificando tanto la posición y orientación de la plataforma base como la configuración articular del robot manipulador. (a)

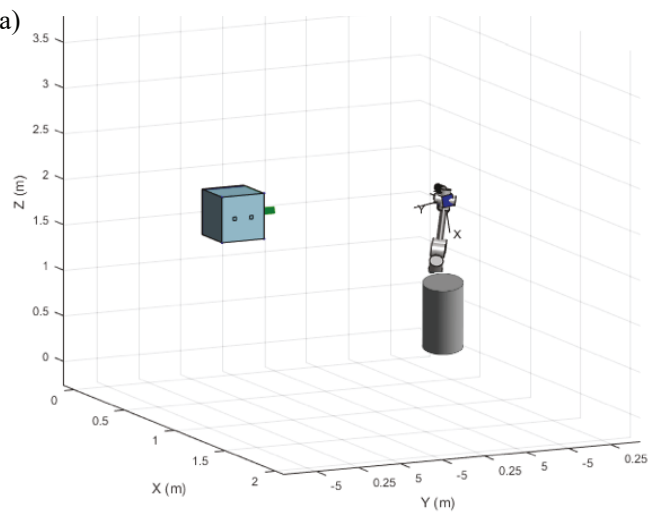

(b)

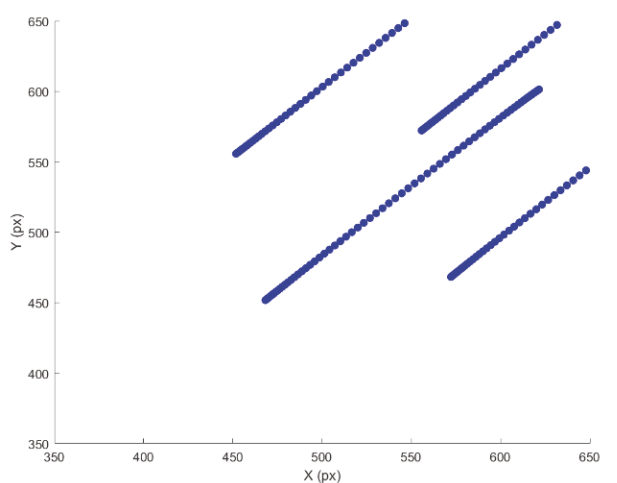

Figura 6: Primer experimento: (a) posición final 3D, (b) trayectoria en la imagen

(a)

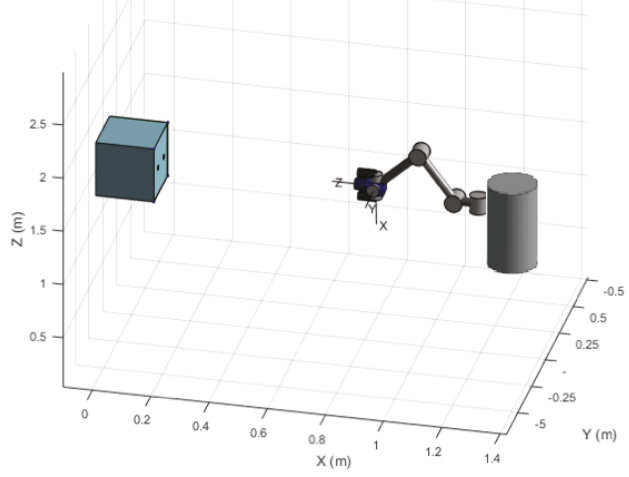

(b)

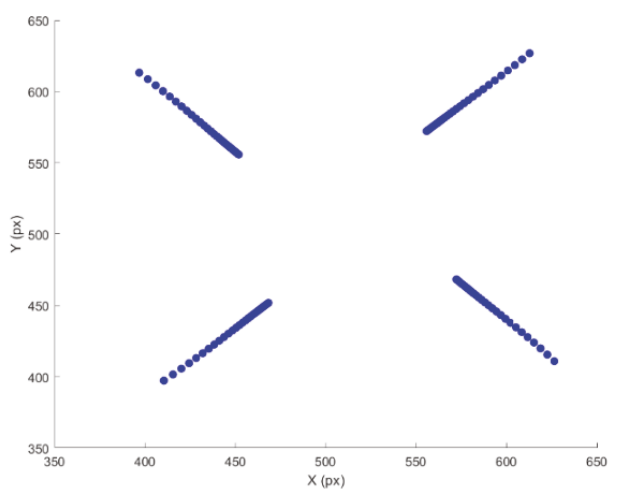

Figura 7: Segundo experimento: (a) posición final $3 \mathrm{D}$, (b) trayectoria en la imagen 


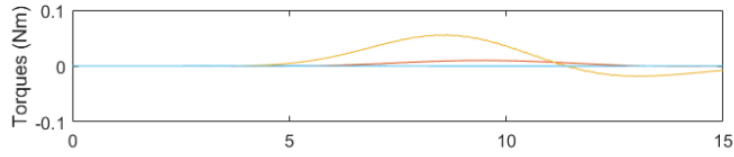

$\mathrm{t}(\mathrm{s})$

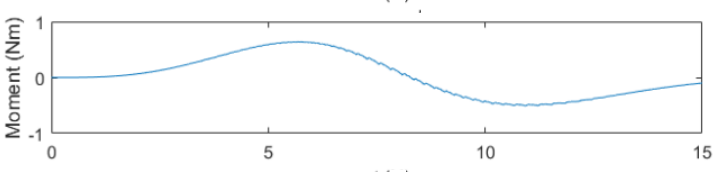

$t(s)$

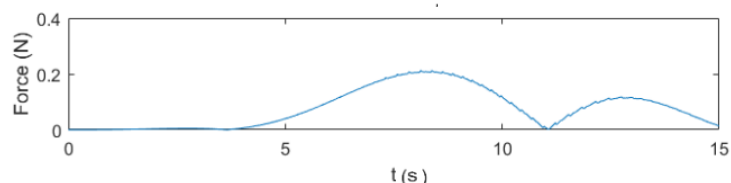

Figura 8: Primer experimento. Par articular, y momento y fuerza aplicados en la plataforma móvil
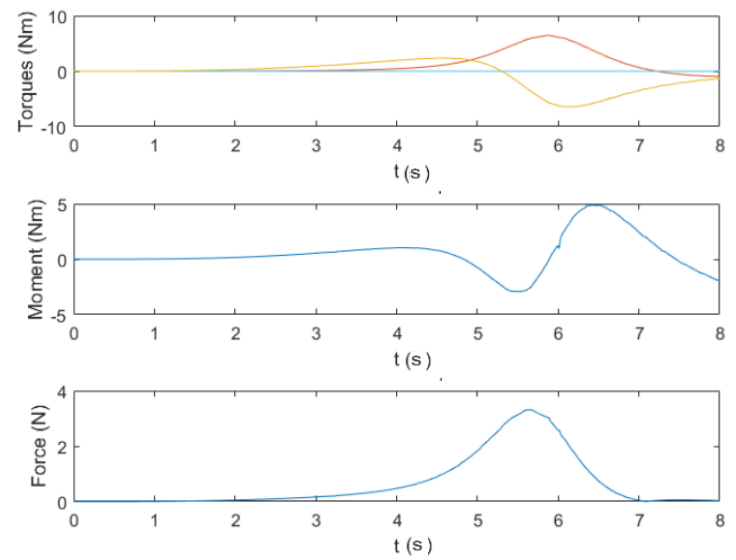

Figura 9: Segundo experimento. Par articular, y momento y fuerza aplicados en la plataforma móvil

\section{CONCLUSIONES}

Este artículo presenta un controlador directo basado en imagen para el guiado de manipuladores móviles. El controlador propuesto considera tanto la realimentación visual como la dinámica del robot para el seguimiento de las trayectorias definidas en la imagen. El controlador genera los pares, la fuerza y los momentos que deben aplicarse al manipulador y la plataforma base para realizar el seguimiento. La realimentación visual se lleva a cabo utilizando un sistema de cámara en configuración eye-in-hand que extrae un conjunto de características visuales del objeto observado.

El controlador se ha probado en una variedad de experimentos, que incluyen aproximaciones a un objeto y tareas de posicionamiento que requieren modificar la posición y/o la orientación del manipulador móvil. En todos estos casos, los experimentos y trayectorias se han realizado con éxito.

\section{Agradecimientos}

Investigación financiada por la Comunidad Valenciana a través del Proyecto GV/2018/050.

\section{English summary}

\section{DYNAMIC CONTROL OF MOBILE MANIPULATORS WITH VISUAL FEEDBACK \\ Abstract}

This paper proposes a direct image-based visual servoing system for the guidance of a mobile manipulator. The robot is composed of a mobile differential platform with a 7 dof (degrees of freedom) arm and an eye-in-hand camera. As described throughout the paper, the visual information is employed to guide both the robot manipulator and the base platform for the tracking of image trajectories. In contrast with previous approaches, the one proposed in this paper employs a direct visual servoing system for guiding mobile manipulators. In this case, the controller directly generates the torques (force and moments) to be applied to the manipulator and base platform taking into account the robot dynamics. This paper describes the different kinematic and dynamic components of the proposed controller and it is validated during the tracking of repetitive and nonrepetitive image trajectories.

Keywords: visual servoing; image-based control; mobile manipulator; direct control

\section{Referencias}

[1] S. Benhimane, and E. Malis, "Homographybased 2D Visual Tracking and Servoing," in The International Journal of Robotics Research, vol. 26, no. 7, pp. 661-676, 2007.

[2] F. Chaumette, and S. Hutchinson, "Visual servo control. I. Basic approaches," in IEEE Robotics $\&$ Automation Magazine, vol. 13, no. 4, pp. 8290, 2006.

[3] A. De Luca, G. Oriolo, and P. R. Giordano, "Image-based visual servoing schemes for nonholonomic mobile manipulators," in Robotica, vol. 25, pp. 131-145, 2007.

[4] O. Khatib, O. (1983). "Dynamic Control of Manipulators in Operational Space". In 6th IFTOMM Congress on Theory of Machines and Mechanisms, pp. 1-10, 1983. 
[5] N. Kumar, J. Borm, V. Panwar, J. Chai, J. "Tracking control of redundant robot manipulators using RBF neural network and an adaptive bound on disturbances". International Journal of Precision Engineering and Manufacturing, vol. 13, no. 8, pp. 1377-1386, 2012.

[6] H. Lang, M. T. Khan, and K. K. Tan, "Application of visual servo control in autonomous mobile rescue robots," in International Journal of Computers Communications \& Control, vol. 11, no. 5, pp. 685-696, 2016.

[7] C. J. Lin. "Motion planning of redundant robots by perturbation method". Mechatronics, vol. 14, no. 3, pp. 281-297, 2004.

[8] J. Nakanishi, R. Cory, M. Mistry, J. Peters, S. Schaal, S. "Operational Space Control: A Theoretical and Empirical Comparison". The International Journal of Robotics Research, vol. 27, no. 6, pp. 737-757, 2008.

[9] J. Pomares, J. A. Corrales, G. J. Garcia, and F. Torres, "Direct visual Servoing to track trajectories in human-robot cooperation," in International Journal of Advanced Robotic Systems, vol. 8, no. 4, pp. 44, 2011.

[10] T. Sandy, and J. Buchli, "Dynamically decoupling base and end-effector motion for mobile manipulation using visual-inertial sensing," in Proceedings of the 2017 IEEE/RSJ
International Conference on Intelligent Robots and Systems (IROS), Vancouver, BC, pp. 62996306, 2017.

[11] E. Tatlicioglu, D. Braganza, T. C. Burg, D. M. Dawson, D. M. "Adaptive control of redundant robot manipulators with sub-task objectives". Robotica, vol. 27, no. 6, pp. 873, 2009.

[12] Y. Wang, G. Zhang, and H. Lang, "A modi ed image- visual servo controller with hybrid camera con guration for robust robotic grasping," in Robotics \& Autonomous Systems, vol. 62, no. 10, pp. 1398-1407, 2014.

[13] B. Xian, M. S. De Queiroz, D. Dawson, I. Walker. "Task-space tracking control of robot manipulators via quaternion feedback". IEEE Transactions on Robotics and Automation, vol. 20, no. 1, pp. 160-167, 2004.

[14] E. Zergeroglu, D. D. Dawson, I. W. Walker, P. Setlur. "Nonlinear tracking control of kinematically redundant robot manipulators". IEEE/ASME Transactions on Mechatronics, vol. 9, no. 1, pp. 129-132, 2004.

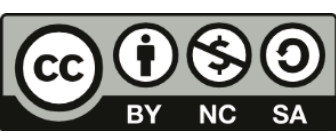

(C) 2019 by the authors. Submitted for possible open access publication under the terms and conditions of the Creative Commons Attribution CC BY-NC-SA 4.0 license (https://creativecommons.org/licenses/bync-sa/4.0/deed.es) 